\title{
BMJ Open Recovery-oriented social work practice in mental health and addictions: a scoping review protocol
}

\author{
Toula Kourgiantakis (D) , ${ }^{1}$ Amina Hussain (D) , ${ }^{1}$ Rachelle Ashcroft (D) , \\ Judith Logan, ${ }^{2}$ Sandra McNeil, ${ }^{1}$ Charmaine C Williams ${ }^{1}$
}

To cite: Kourgiantakis T, Hussain A, Ashcroft R, et al. Recovery-oriented social work practice in mental health and addictions: a scoping review protocol. BMJ Open 2020;10:e037777. doi:10.1136/ bmjopen-2020-037777

- Prepublication history and additional material for this paper are available online. To view these files, please visit the journal online (http://dx.doi. org/10.1136/bmjopen-2020 037777).

Received 15 February 2020

Revised 15 July 2020

Accepted 16 July 2020
Check for updates

(C) Author(s) (or their employer(s)) 2020. Re-use permitted under CC BY-NC. No commercial re-use. See rights and permissions. Published by BMJ.

${ }^{1}$ Factor-Inwentash Faculty of Social Work, University of Toronto, Toronto, Ontario, Canada

2John P. Robarts Library, University of Toronto, Toronto, Ontario, Canada

Correspondence to Dr Toula Kourgiantakis; toula.kourgiantakis@utoronto.ca

\section{ABSTRACT}

Introduction Social work is a key profession in the field of mental health worldwide and the profession has values that are aligned with a recovery paradigm. However, there are gaps in understanding how social workers are applying the recovery paradigm in practice. This study will scope and synthesise the literature related to recovery and social work practice in mental health and addictions. There will also be an exploration of best practices and gaps in recovery-oriented social work practice.

Methods and analysis Using a scoping review framework developed by Arksey and 0'Malley, we will conduct our search in five academic databases: PsycINF0, Medline, CINAHL Plus, Sociological Abstracts and Social Services Abstracts. Articles meeting inclusion criteria will be charted to extract relevant themes and analysed using a qualitative thematic analysis approach.

Ethics and dissemination This review will provide relevant information about best practices and gaps in recovery-oriented social work practice in mental health and addictions. The study will inform the development of mental health curricula in social work programmes and clinical settings. Results will be disseminated through a peer-reviewed journal and at conferences focusing on mental health, addictions, and social work education. Ethics approval is not required for this scoping review.

\section{INTRODUCTION}

Recovery is a paradigm with increasing influence on mental health systems and policies in many high-income countries over the last two decades, ${ }^{1-3}$ and it is included in the WHO's Mental Health Action Plan. The recovery paradigm was introduced in the 1980 s by mental health consumers ${ }^{5}$ as an alternative to the biomedical model focusing on illness, chronicity, and cure. ${ }^{6}$ We will use the term paradigm defined by Kuhn as a 'constellation of beliefs, values, techniques, and so on shared by the members of a given community' (p175). Members of a community share assumptions and beliefs, practice using a specific paradigm and pursue common goals. Practice interventions and theories are developed and shaped by paradigms. ${ }^{89}$ Recovery has been defined as 'a way

\section{Strengths and limitations of this study}

This is the first comprehensive review of the recovery paradigm and social work practice in mental health and addictions.

- The search strategy has been developed by a research team with expertise in the methodology and subject area.

- Due to the nature of the scoping review framework, the studies included in the review will not be appraised for quality.

- This scoping review will include all article types and methodologies but will not include books or grey literature.

of living a satisfying, hopeful, and contributing life even with limitations caused by illness ${ }^{9}$ (p15). Researchers in the UK developed a CHIME framework which refers to recovery-oriented processes that include connectedness, hope, identity, meaning, and empowerment. ${ }^{10}$ Other countries have adopted common guiding principles for recovery that include CHIME concepts and other important processes related to recovery: (1) hope, (2) lived experiences, (3) individual, family and community strengths, (4) self-determination, (5) peer support, (6) collaborative relationships, (7) a non-linear process, (8) a holistic approach, (9) cultural diversity, and (10) social inclusion, stigma and discrimination. ${ }^{11-13}$

A similar movement towards a recovery framework has been adopted for addictionrelated concerns which include substance use and behavioural addictions. ${ }^{11}{ }^{1314}$ While the services and approaches to treatment may be different for mental health concerns than they are for those coping with addictions, there are similar and overlapping principles of recovery. ${ }^{1115}$ For some individuals coping with an addiction-related concern, the pathway to recovery may involve abstinence, while for others, it is about reducing the 
harm. ${ }^{14} \mathrm{~A}$ term that is specific to addictions is recovery capital, which refers to 'the many resources one can use towards their recovery from alcohol and other drug dependency $^{16}$ (p.349). Mental health and addiction services have a longstanding history of being divided in terms of policies and service provisions but a review of recovery-oriented practice guidelines indicate that there are overlapping values and guiding principles related to recovery-oriented care and a 'need for a unified vision of well-being ${ }^{, 17}$ (p.12).

Although many countries such as Canada have adopted a recovery framework for their national mental health strategy, researchers report challenges for mental healthcare professionals to implement recovery principles in practice and the culture of many systems of mental healthcare does not reflect a recovery paradigm. ${ }^{23}$ The social work profession has a longstanding history of important and unique contributions in the field of mental health ${ }^{18} 19$ and addictions. ${ }^{20}$ The WHO identifies social work as a key profession in mental health across 149 countries. ${ }^{4}$ An American survey found that social workers' most common speciality practice area is mental health and most social workers engage with individuals and families with mental health concerns even when working outside of this specific field. Irrespective of their practice domain, most social workers support clients with mental illness (96\%), and addictions concern $(87 \%) .{ }^{21}$ The recovery paradigm is strongly aligned with social work values and conceptual frameworks promoting empowerment, partnership, and choice informed by ecosystems theory and a strength-based model. ${ }^{6} 2223$ Despite social work's unique alignment with the recovery paradigm, researchers argue that social work has not had a strong voice in challenging and critiquing the dominant biomedical model. ${ }^{23}{ }^{24}$ There are gaps in understanding the extent to which social workers are applying recovery guiding principles in practice. ${ }^{24}{ }^{25}$ Researchers have identified several impediments to implementation, including the lack of a universal definition of recovery-oriented care and a paucity of evidence-based research to inform practice. ${ }^{24-26}$ The organisational context may also influence implementation by pressuring social workers to adhere to institutional policies and procedures that may be incongruent with recovery principles. ${ }^{24}$

Williams and colleagues ${ }^{26}$ argue that recovery does not adequately address sociopolitical issues related to power and control over mental healthcare. Social work's core value of social justice can make valuable contributions to advancing how recovery is implemented in mental healthcare systems; however, social work has also been critiqued for its conformity with dominant structural systems that are not recovery oriented and perpetuate stigma and discrimination. ${ }^{26}$ Considering the important role of social workers internationally, we need a greater understanding of how social workers are conceptualising and implementing recovery in mental health and addictions. ${ }^{13}$ Moreover, research has shown that recoveryoriented practice is ambiguous and it is important for clinicians to learn to operationalise this concept and guidelines needed that are context specific. ${ }^{27}$ There is a lack of guidelines for clinical application of recoveryoriented care and attempts to operationalise this have been through the lens of organisational priorities. ${ }^{28}$

This paper delineates a protocol for a scoping review on the recovery paradigm in social work in mental health and addictions. The objectives of this review are to (1) scope the literature related to the recovery paradigm in social work in mental health and addictions, (2) synthesise definitions, principles and values related to recoveryoriented social work practice in mental and addictions, (3) describe how recovery is implemented in social work practice, and (4) identify evidence-based practices and gaps in recovery-oriented social work practice in mental health and addictions.

\section{METHODS AND ANALYSIS}

A scoping review will be conducted to map existing literature on recovery-oriented practice within social work education, research and practice in mental health and addictions. Scoping reviews involve systematically mapping recurring themes, concepts and identifying recommendations from the current literature as they relate to the research question at hand. ${ }^{29}$ This study will employ the scoping review framework espoused by Arksey and $\mathrm{O}^{\prime}$ Malley ${ }^{30}$ that consists of five stages: (1) identifying the research question, (2) identifying relevant studies, (3) study selection, (4) charting the data, and (5) collating, summarising and reporting the results. Given the nature of this exploratory study, this form of knowledge synthesis will be valuable in providing a breadth of literature pertaining to the recovery paradigm within social work education, research and practice. We will adhere to the Preferred Reporting Items for Systematic Review and Meta-Analysis (PRISMA) Extension for Scoping Reviews (PRISMA-ScR) reporting guidelines. ${ }^{31}$ See online supplementary appendix A for the PRISMA-ScR checklist.

\section{Stage 1: identifying the research questions}

As highlighted throughout our literature review, recovery is central to social work practice and there have been no studies charting the evidence on the recovery paradigm in social work education, research, and practice in mental health and addictions. Based on recommendations by Colquhoun $e t a l^{32}$ the research questions for this scoping review were developed collaboratively by our research team consisting of three social work faculty members (TK, RA, and CCW), one social science librarian (JL) and two social work doctoral students (AH and SM).

The research team developed the following research questions: (1) How is the recovery-paradigm conceptualised and defined in social work practice in mental health and addictions? (2) What are the principles and values of recovery in social work? (3) How is the recovery paradigm used in social work practice, education and research? (4) What are the gaps, challenges or barriers of recovery 


\begin{tabular}{|c|c|}
\hline Number & Searches \\
\hline 1 & social work*.tw. \\
\hline 2 & casework $^{\star}$.tw. \\
\hline 3 & case work ${ }^{*}$.tw. \\
\hline 4 & social workers/ \\
\hline 5 & psychiatric social workers/ \\
\hline 6 & Social case work/ \\
\hline 7 & or/1-6 [social work) \\
\hline 8 & $\begin{array}{l}\text { (recover }^{*} \text { adj3 } \text { (care or practic }^{*} \text { or educat }{ }^{*} \text { or curricul } \\
\text { or teach* }^{*} \text { or learn* or train* or research* or therap* or } \\
\text { support }{ }^{*} \text { or orient }{ }^{*} \text { or approach }{ }^{\star} \text { or model? or health or } \\
\left.\text { mental health or institutional or capital or natural }{ }^{*}\right) \text { ).tw. }\end{array}$ \\
\hline 9 & "recovery (disorders)"/ \\
\hline 10 & exp Rehabilitation/ \\
\hline 11 & recover*.tw. \\
\hline 12 & 10 and 11 \\
\hline 13 & 8 or 9 or 12 \\
\hline 14 & 7 and 13 \\
\hline
\end{tabular}

in social work? (5) What are the recommendations, evidence-based or best practices for using a recovery paradigm in social work research, education and practice?

\section{Stage 2: identifying relevant studies}

The initial search strategy was developed in PsycINFO (Ovid, 1806-) by the social sciences librarian (JL) in consultation with other team members. See table 1 for the draft search strategy in PsycINFO. It will be sent to a second librarian for peer review, using the Peer Review of Electronic Search Strategies framework. ${ }^{33}$ Any subsequent feedback will be incorporated to enhance the breadth and scope of articles generated from the search. Our search strategy will be conducted in five academic databases: PsycINFO (1806-), Medline (1946 -), CINAHL Plus (1937-), Sociological Abstracts (1952-) and Social Services Abstracts (1979-). These databases were intentionally selected for their inclusion of mental health literature as well as research on social work practice and education, and thus are likely to capture relevant scholarly material. Furthermore, we will conduct a citation search of the reference lists of selected articles to ensure that a wider scope of articles are included.

\section{Stage 3: study selection}

After generating a list of articles from our search strategy, we will engage in an iterative, peer-review two-stage screening process with two independent reviewers at each stage. In the first stage, two independent reviewers will screen articles for suitability based on their title and abstracts. In the second stage, the reviewers will independently conduct a full-text review of the selected articles to ensure their content meets our inclusion criteria outlined below. If there is ambiguity on whether certain articles fit the scope of this protocol, a third reviewer will be consulted. We will use Covidence- $\mathrm{a}$ web-based software for systematic and scoping reviews that facilitates screening, study selection and data extraction. ${ }^{34}$ This protocol will focus on articles written in English only and selected material will include empirical studies, literature reviews, dissertations, teaching articles and conceptual/ theoretical papers. There are no outlined geographical or date restrictions. Articles must meet the following inclusion criteria to be selected: (1) include the term recovery in the title or abstract, (2) use the term social work or social worker in the title or abstract, (3) have an explicit focus on recovery that may include different concepts such as recovery, recovery model, recovery-oriented practice, recovery-oriented care and so on, (4) explicitly focus on social work research, education, training or practice in relationship to recovery, and (5) focus on recovery with respect to mental health and/or addictions. We will exclude books, book reviews, editorials and grey literature.

\section{Stage 4: Charting the data}

Key themes extracted from the selected articles will be categorised, summarised and presented clearly within a data charting form. The research team developed initial charting variables based on the research questions and these variables will be used to extract data and identify key themes from selected articles. The preliminary variables that will be used to categorise information include (1) authors, (2) year, (3) country where study was conducted or country of first author's affiliation, (4) journal, (5) format of paper (empirical, literature review, dissertation, conceptual/theoretical, editorial, teaching article, etc), (6) definition of recovery, (7) principles of recovery, (8) area of focus (eg, mental health, addictions, etc), (9) implementation of recovery, (10) targeted audience (eg, students, service users, social workers, etc), (11) focus on equity or access, (12) focus on stigma, (13) focus on race, culture and/or diversity, (14) gaps, challenges or barriers, and (15) recommendations, evidence-based or best practices. To assess whether these headings accurately capture the scope and breadth of the content, the reviewers will have two research assistants independently chart the first five articles that meet our inclusion criteria, and if necessary, refine the definitions for the variables/charting categories. We will also engage in a constant comparative method and peer review to minimise any discrepancies during the charting process. The researchers will also engage in a qualitative thematic analysis to identify and highlight themes present among this chart. The charted data will be organised and presented in a Microsoft Excel spreadsheet.

\section{Stage 5: identification, synthesis and report of study findings}

Findings outlined in the charted data will be reviewed, synthesised and analysed through a numerical summary analysis as well as a qualitative thematic analysis. The final scoping review will be presented in publications and at upcoming conferences. Study findings will be disseminated to relevant stakeholders such as researchers, clinicians and social work educators. Anticipated findings are expected to map out the current nature and scope of recovery in social work practice in mental health and addictions, and the scoping review will 
provide recommendations for recovery-oriented social work practice in mental health and addictions.

\section{Patients and public involvement}

Neither patients nor members of the public were involved in this project.

\section{ETHICS AND DISSEMINATION}

The scoping review outlined in this paper contributes to our current understanding and will advance knowledge of recovery-oriented social work practice in mental health and addictions. The information gathered for this paper and the outlined scoping review were retrieved from publicly available sources, therefore ethics approval is not required for this project. The results will be disseminated through a peerreviewed journal and reported at national and international conferences on mental health and addictions as well as social work education, practice, and research.

\section{Twitter Toula Kourgiantakis @DrKourgiantakis}

Contributors All authors made substantive intellectual contributions to the development of this protocol. TK and AH developed, wrote and edited the initial protocol. JL developed the search strategy and contributed to the writing of the protocol. All authors (TK, AH, JL, SM, RA and CCW) critically reviewed and revised the final version prior to submission.

Funding This scoping review protocol is supported by a 2019-2020 Royal Bank of Canada Graduate Fellowship in Applied Social Work Research, Factor-Inwentash Faculty of Social Work, University of Toronto.

Competing interests None declared.

Patient and public involvement Patients and/or the public were not involved in the design, or conduct, or reporting, or dissemination plans of this research.

Patient consent for publication Not required.

Provenance and peer review Not commissioned; externally peer reviewed.

Open access This is an open access article distributed in accordance with the Creative Commons Attribution Non Commercial (CC BY-NC 4.0) license, which permits others to distribute, remix, adapt, build upon this work non-commercially, and license their derivative works on different terms, provided the original work is properly cited, appropriate credit is given, any changes made indicated, and the use is non-commercial. See: http://creativecommons.org/licenses/by-nc/4.0/.

\section{ORCID iDs}

Toula Kourgiantakis http://orcid.org/0000-0002-2491-2595

Amina Hussain http://orcid.org/0000-0002-8697-0360

Rachelle Ashcroft http://orcid.org/0000-0002-5666-1946

\section{REFERENCES}

1 Adams N, Daniels A, Compagni A. International pathways to mental health transformation. Int J Ment Health 2009;38:30-45.

2 Kidd SA, Mckenzie KJ, Virdee G. Mental health reform at a systems level: widening the lens on recovery-oriented care. Can J Psychiatry 2014;59:243-9.

3 Piat M, Sabetti J. The development of a recovery-oriented mental health system in Canada: what the experience of Commonwealth countries tells us. Can J Commun Ment Health 2009;28:17-33.

4 WHO. Mental health action plan 2013-2020. Geneva: World Health Organization, 2013.

5 Deegan PE. Recovery: the lived experience of rehabilitation. $J$ Rehabil Psychosoc 1988;11:11-19.

6 Carpenter J. Mental health recovery paradigm: implications for social work. Health Soc Work 2002;27:86-94.
7 Kuhn TS. The structure of scientific revolutions. University of Chicago press, 2012.

8 Ashcroft R, Van Katwyk T, Hogarth K. An examination of the Holism paradigm: a view of social work. Soc Work Public Health 2017;32:461-74

9 Anthony WA. Recovery from mental illness: the guiding vision of the mental health service system in the 1990s. J Psychosoc Rehabil 1993;16:11-23.

10 Leamy M, Bird V, Le Boutillier C, et al. Conceptual framework for personal recovery in mental health: systematic review and narrative synthesis. Br J Psychiatry 2011;199:445-52.

11 Mental Health Commission of Canada. Guidelines for recoveryoriented practice. Ottawa: Mental Health Commission of Canada, 2015.

12 Commonwealth of Australia. A national framework for recoveryoriented mental health services: policy and theory, 2013.

13 Substance Abuse and Mental Health Services Administration (SAMHSA). SAMHSA'S working definition of recovery: 10 guiding principles of recovery. Department of Health and Human Services, 2012: 1-7.

14 Canadian Centre on Substance Use and Addiction. Moving toward a Recovery-Oriented system of care: a resource for service providers and decision makers, 2017. Available: https://www.ccsa.ca/sites/ default/files/2019-04/CCSA-Recovery-Oriented-System-of-CareResource-2017-en.pdf

15 Davidson G, Brophy L, Campbell J. Risk, recovery and capacity: competing or complementary approaches to mental health social work. Aust Soc Work 2016;69:158-68.

16 Hennessy EA. Recovery capital: a systematic review of the literature. Addict Res Theory 2017;25:349-60.

17 Bartram M. 'It's really about wellbeing': a Canadian investigation of harm reduction as a bridge between mental health and addiction recovery. Int J Ment Health Addict 2020;20.

18 Ashcroft R, Van Katwyk T. Joining the global conversation: social workers define health using a participatory action research approach Br J Soc Work 2017;47:bcw005.

19 Ashcroft R, Kourgiantakis T, Fearing G, et al. Social work's scope of practice in primary mental health care: a scoping review. Br J Soc Work 2019;49:318-34.

20 Straussner SLA. The role of social workers in the treatment of addictions. J Soc Work Pract Addict 2001;1:3-9.

21 Whitaker T, Weismiller T, Clark E. Assuring the sufficiency of a frontline workforce: a national study of licensed social workers, 2006.

22 Davidson L, White W. The concept of recovery as an organizing principle for integrating mental health and addiction services. $J$ Behav Health Serv Res 2007;34:109-20.

23 Hyde B, Bowles W, Pawar M. 'We're Still in There'-consumer voices on mental health inpatient care: social work research highlighting Lessons for recovery Practice: Table 1. Br J Soc Work 2015;45:i62-78.

24 Khoury E, Rodriguez del Barrio L. Recovery-oriented mental health practice: a social work perspective. Br J Soc Work 2015;45:i27-44

25 Loumpa V. Promoting recovery through peer support: possibilities for social work practice. Soc Work Health Care 2012;51:53-65.

26 Williams CC, Almeida M, Knyahnytska Y. Towards a biopsychosociopolitical frame for recovery in the context of mental illness. Br J Soc Work 2015;45:i9-26.

27 Le Boutillier C, Leamy M, Bird VJ, et al. What does recovery mean in practice? A qualitative analysis of international recovery-oriented practice guidance. Psychiatr Serv 2011;62:1470-6.

28 Le Boutillier C, Chevalier A, Lawrence V, et al. Staff understanding of recovery-orientated mental health practice: a systematic review and narrative synthesis. Implement Sci 2015;10:87.

29 Levac D, Colquhoun H, O'Brien KK. Scoping studies: advancing the methodology. Implement Sci 2010;5:69.

30 Arksey H, O'Malley L. Scoping studies: towards a methodological framework. Int J Soc Res Methodol 2005;8:19-32.

31 Tricco AC, Lillie E, Zarin W, et al. PRISMA extension for scoping reviews (PRISMA-ScR): checklist and explanation. Ann Intern Med 2018; $169: 467-73$

32 Colquhoun HL, Levac D, O'Brien KK, et al. Scoping reviews: time for clarity in definition, methods, and reporting. J Clin Epidemiol 2014;67:1291-4.

33 McGowan J, Sampson M, Salzwedel DM, et al. PRESS peer review of electronic search strategies: 2015 guideline statement. J Clin Epidemiol 2016;75:40-6.

34 Macdonald M, Martin Misener R, Weeks L, et al. Covidence vs Excel for the title and abstract review stage of a systematic review. Int $J$ Evid Based Healthc 2016;14:200-1. 